\title{
Strates
}

STRATES Matériaux pour la recherche en sciences sociales

9 | 1997

Crises et mutations des territoires

\section{Pour une nouvelle approche spatiale de l'exclusion sociale}

Nicole Mathieu

\section{(2) OpenEdition}

1 Journals

Édition électronique

URL : http://journals.openedition.org/strates/612

DOI : $10.4000 /$ strates. 612

ISSN : $1777-5442$

\section{Éditeur}

Laboratoire Ladyss

\section{Édition imprimée}

Date de publication : 30 septembre 1997

ISSN : 0768-8067

\section{Référence électronique}

Nicole Mathieu, «Pour une nouvelle approche spatiale de l'exclusion sociale », Strates [En ligne], 9 | 1997, mis en ligne le 19 octobre 2005, consulté le 08 septembre 2020. URL : http://

journals.openedition.org/strates/612; DOI : https://doi.org/10.4000/strates.612

Ce document a été généré automatiquement le 8 septembre 2020.

Tous droits réservés 


\title{
Pour une nouvelle approche spatiale de l'exclusion sociale
}

\author{
Nicole Mathieu
}

1 Avec la lancinante montée dans les médias et dans les déclarations politiques de tous bords du discours sur l'exclusion, avec la progression organisée des revendications pour le rétablissement des «droits sociaux fondamentaux » par l'accès à l'emploi, au logement et à des «ressources financières décentes $»^{1}$, avec, en co-occurrence, le discours, également lancinant, de l'immigration, des «banlieues » et des «territoires de l'exclusion $»^{2}$, les scientifiques peuvent difficilement ne pas s'impliquer aujourd'hui en mobilisant, sur la question de l'exclusion, le mode théorique qui leur est propre.

2 Cette nécessité s'est déjà imposée à certains, en particulier à des sociologues qui se sont engagés, chacun à leur manière, dans la compréhension de ce retour aux termes populaires de misère et de "miséreux », de la résurgence des "sans travail », de la mendicité et de l'errance. Mais, comme Chantal Balley le signale dans son éditorial du Géoforum 1993 (Balley, 1994), les géographes ont abordé avec parcimonie le thème des exclusions et des pauvretés dans leurs dimensions récentes. Une question devait orienter les débats croisés : «Quelle est la place du territoire dans le traitement des situations de pauvreté ?» Elle est me semble-t-il restée presque entièrement en friche. Certes, les travaux de géographes portant en particulier sur le RMI se sont développés. Mais ils n'ont pas été au fond de ce qui, de mon point de vue, fait vraiment problème pour les sciences sociales et concerne tout particulièrement la géographie : comment penser les rapports entre ces deux termes: exclusion et territoire? Les théories existantes nous permettent-elles d'analyser les phénomènes dits d'exclusion aujourd'hui ? Comment mener nos recherches pour qu'elles soient utiles à ceux qui revendiquent ce droit à une égalité minimum? Le texte qui suit a pour objectif de contribuer à cette réflexion. C'est à l'occasion d'une recherche effectuée pour le Commissariat Général au Plan (STRATES/SEGESA, 1996) qu'il a été élaboré mais, comme cela se dit, et surtout, parce qu'il ne repose que sur des lectures et une expérience partielle (plutôt en milieu rural), il n'engage que la responsabilité de l'auteur, appelant 
la discussion et la controverse jusqu'à ce que les questions posées trouvent une réponse.

La place de l'espace dans le thème de l'exclusion : entre surdétermination et sousestimationEspace et pauvreté, deux phénomènes pensés interdépendants

3 Une relecture personnelle et sommaire des travaux le plus souvent cités sur le thème de la pauvreté et de l'exclusion et qui sont antérieurs à la crise actuelle, montre que, pourtant, en sociologie comme en géographie, une place essentielle est traditionnellement accordée à l'espace, au local et au territoire. L'ensemble de ces recherches se rattachent, parfois en les contestant, aux théories des sociologues de l'école de Chicago élaborées dans les années trente. A travers le concept d'espace social, ces recherches interrogent les rapports entre les spécificités de l'espace géographique intra-urbain (le centre, la périphérie, la banlieue...) et les identités sociales de ses habitants (bourgeoisie, cols blancs, ouvriers, étrangers, immigrés, races et ethnies...) Dans le prolongement de cette orientation, un courant de recherche désigné « écologie urbaine" a proposé de distinguer des espaces urbains, des quartiers, dont l'identification repose essentiellement sur le caractère dominant de leur population, d'où les dénominations comme quartiers (de) riches, quartiers (de) pauvres, quartiers ethniques (italiens, asiatiques, noirs, etc.). Dans certains travaux sont aussi soulignés, à côté des critères proprement spatiaux (distance au centre, nodalité, accessibilité), le rôle de critères de milieu qui déterminent la localisation des habitats de "prolétaires " (espace sous le vent d'ouest de Saint-Denis où sont localisées les industries "polluantes» et les cités des ouvriers qui y travaillent), ou de "pauvres» (favelas brésiliennes localisées dans des terrains en pente ou inondables, inaccessibles et à risques, etc.). En somme à la marginalité sociale correspondrait une marginalité spatiale.

4 Parfois statiques lorsqu'elles privilégient la mise en évidence des structures spatiales organisant la ville, ces recherches, en approfondissant les rapports entre dynamiques sociales et dynamiques spatiales, entre marginalisation sociale et marginalisation spatiale, ont le plus souvent abouti à l'identification de processus socio-spatiaux que la notion de "ségrégation dans la ville » synthétise. En mettant en évidence l'existence de dynamiques de dévalorisation/revalorisation de quartiers et d'îlots urbains, sur la base de changement des valeurs foncières et des loyers mais aussi de changements des représentations culturelles et des normes sociales de l'habitat, ces travaux ont montré le double mouvement de "gentrification » et de "paupérisation» suivant deux modèles spatiaux principaux ${ }^{3}$ : le modèle nord-américain où le centre se déqualifie jusqu'à la violence et le "ghetto » tandis que les périphéries s'embourgeoisent; le modèle européen où les quartiers centraux où l'habitat ancien est rénové et la construction neuve de qualité sont reconquis par la "gentry » tandis que les banlieues et les espaces marqués par les grands ensembles et l'habitat social deviennent les espaces des nouvelles « classes dangereuses» et de l'« ethnicisation». Certes le terme de "ségrégation dans la ville " a été largement discuté (Brun, Rhein, 1994) jusqu'à s'interroger sur sa pertinence par rapport à celle de "division sociale de l'espace » (Rhein, 1994). Mais ces recherches, en dépit de la diversité des postures théoriques, s'appuient sur un paradigme selon lequel la dynamique sociale, et en particulier le processus d'exclusion, ne peut être dissocié des processus territoriaux, les deux logiques étant actives et interactives, ce qui conduit à une accentuation, à un renforcement des ségrégations et sociales et spatiales. 
Exclusion territoriale, exclusion sociale, une interdépendance limitée

Il ne s'agit pas de mettre en cause cette forme de théorisation des rapports entre sociétés et territoires qui a produit et produit encore des recherches de tout premier ordre pour la compréhension et le traitement des processus ségrégatifs. Ce qui m'importe est de dénoncer l'usage stéréotypé qui en a été fait et les dérives qu'elle a engendrées par surdétermination du facteur spatial dans l'appréhension des processus (nouveaux) de l'exclusion sociale.

6 La première dérive provient précisément de l'attitude critique (de géographie critique ou radicale) que suscite le constat de l'« injustice spatiale » et des discriminations des territoires les uns par rapport aux autres. Celle-ci a eu pour conséquence d'utiliser le terme de "territoire d'exclusion", "en particulier pour désigner des "bouts" de ville, des quartiers, où la concentration de population en voie de désinsertion économique et sociale est $s i$ marquée qu'en quelque sorte elle les spécialise " (Jaillet, 1994). Outre le fait que cette désignation occulte une réalité socio-économique plus complexe et diversifiée que les descripteurs permettant de définir un « territoire d'exclusion » ne le laissent supposer, elle a surtout conduit, dans le discours dominant, à un amalgame entre exclusion sociale et exclusion territoriale et à un recours systématique au territoire comme mode d'opérationalisation des politiques publiques contre l'exclusion. On comprend certes les enjeux qui peuvent motiver ce recours au territoire dans les politiques publiques contre la «fracture sociale» ou l'«exclusion»: relance de la politique d'État de correction des inégalités, nécessité d'obtenir la mobilisation de l'échelon local dans la mise en œuvre des politiques de lutte contre l'exclusion, avantage de la transversalité $\mathrm{du}$ territoire permettant d'articuler les politiques sectorielles et donc de les évaluer, etc. Mais l'association systématique qui est faite entre territoires et exclusion, pour les banlieues en particulier, constitue un obstacle à l'identification et à la compréhension fine des mécanismes actuels de l'exclusion, conduit à des stigmatisations et des présupposés (l'identité ou l'enfermement des exclus dans leurs territoires), n'apporte aucune garantie sur l'« adéquation entre la circonscription des maux et des remèdes » (Jaillet, 1994).

7 A l'inverse, la deuxième dérive consiste à sous-estimer certains types d'espaces et de territoires, comme les espaces ruraux, parce qu'il ne s'y produit pas de spécialisations fortes entre catégories sociales et espaces et parce que les phénomènes de pauvreté y sont par nature plus diffus. Les discours politiques et médiatiques amalgament ainsi sans discernement exclusion et quartiers défavorisés ou ethniques, mais ils ignorent la localisation de phénomènes mobiles ou invisibles de l'exclusion dans le tissu urbain (progression de la mendicité dans les centre-villes, les espaces de transport public, les espaces commerciaux; extension des squats et des «stations» de SDF). Ainsi, dans l'idéologie dominante, il est impossible d'imaginer que la pauvreté, le chômage, la précarité et l'exclusion puissent se développer en milieu rural dans la mesure où elle ne prend pas le visage conforme aux stéréotypes (immigration, étrangers, drogue, délinquance...) et que la capacité de "solidarité » de l'espace rural est surestimée, sous prétexte qu'il s'agit d'un espace de l'inter-connaissance et de la « communauté » ${ }^{4}$. L'absence du territoire dans la problématique sociologique actuelle de l'exclusion Ce qui frappe, inversement, c'est l'absence de tout recours au territoire dans les recherches sociologiques actuelles traitant de la question de l'exclusion (Paugam, 1996). 
9 Avec l'aggravation du chômage, des jeunes en particulier, avec l'accentuation de la précarisation des emplois, le développement d'une pauvreté visible et de manifestations violentes proches des jacqueries d'autrefois, le thème de la «nouvelle pauvreté » et de l'exclusion est devenu central dans les recherches. Les travaux se sont multipliés autour de questions : pourquoi le terme d'exclusion envahissait-il la scène sociale? s'agissait-il d'un symptôme ou d'un phénomène social? désignait-il des phénomènes en continuité avec ceux observés antérieurement? ou au contraire des faits nouveaux traduisant des ruptures économiques et sociales? l'exclusion était-elle irréversible ou réversible par l'insertion? quels groupes étaient les plus vulnérables? la formation était-elle un rempart? Toutes ces interrogations ont suscité la mobilisation de nombreux groupes de recherche et la réalisation d'enquêtes pour mieux comprendre et interpréter ces formes nouvelles du changement social.

En fait l'effort de recherche a essentiellement porté sur les dimensions sociologiques et psychologiques du phénomène de l'exclusion et des processus qui y conduisent. On a d'abord mis en évidence la diversité des trajectoires et des situations qui engendraient l'exclusion du travail. La catégorie des jeunes a été particulièrement travaillée ainsi que le rapport entre formation et insertion professionnelle. La connaissance de nouvelles catégories d'« exclus » a aussi progressé, telle par exemple celle des Sans Domicile Fixe attirant l'attention sur des facteurs d'exclusion moins connus que le chômage comme l'incidence du logement, de l'absence de soutien familial etc. La relation entre niveau de formation et accession privilégiée à l'emploi a été remise en cause avec le développement du chômage des diplômés et des cadres.

11 La théorisation des processus qui conduisent à l'exclusion sociale a incontestablement progressé parallèlement aux controverses scientifiques sur la caractérisation de ce changement social : critique de la théorie de la reproduction des classes et donc de la reproduction des "pauvres", théorie de la disqualification sociale ou de la désaffiliation insistant sur la perte d'identité des individus et de lien social, interprétation du développement de mouvements d'auto-exclusion (communautés, sectes) comme une réaction à la régression sociale, etc.

Plus récemment et parallèlement à la montée des critiques contre l'abus de la terminologie de l'exclusion (Joint-Lambert, 1995), les recherches ont mis l'accent sur la question de la définition du concept lui-même (Moreau de Bellaing, 1996), sur l'émergence récente des termes d'exclu et d'exclusion, ainsi que sur l'évolution des représentations du pauvre et de l'exclus dans le temps, variation qui dépend "étroitement des grands problèmes qui se posent à la société dans son ensemble, à une période donnée » (Sassier, 1996).

13 La sociologie, parce qu'elle se définit comme une démarche d'élucidation du fait social (du «fait social total»), est devenue la discipline centrale des investigations de l'exclusion. Ainsi Moreau de Bellaing propose de définir l'exclusion non seulement comme un "fait social d'excès » mais aussi comme un processus d'excès se glissant dans les processus sociaux (l'exclusion transgresse les droits) ${ }^{5}$. Pour lui l'exclusion implique des ruptures, celle entre travailleurs et chômeurs - le chômage de longue durée menant à la fin des droits et donc à la grande pauvreté et éventuellement à la misère -, celle entre hommes et femmes, entre individus en bonne santé physique et mentale et handicapés physiques et mentaux, celle entre intégrés, assimilés, socialisés et exclus (pauvres, nouveaux pauvres, grands pauvres comme les déracinés et les migrants, misérables), enfin entre les différentes catégories de pauvres et les 
misérables. A la lumière de la chronique du salariat de Robert Castel (Castel, 1995), on peut se demander si une rupture ne s'instaure pas dans les représentations et les normes du travail.

Le travail social comme seul recours pour lutter contre l'exclusion

14 Mais cette posture théorique conduit à ne privilégier que l'approche individuelle et sociale quand il s'agit de lutter contre l'exclusion par l'insertion. Qu'il s'agisse du travail de retour à l'emploi des exclus, du travail d'insertion et de réinsertion, du travail de réidentification et de resocialisation, du renforcement des défenses collectives et individuelles contre les abus et la transgression des droits et des obligations, qu'il s'agisse de la reculturation, tout ce travail politique n'est pensé que comme un travail social fait par des institutions (service social, institution psychiatrique, psychologie clinique...) et des associations de tous types (depuis l'aide alimentaire jusqu'au chantier d'insertion) en direction d'individus considérés comme des individualités et de groupes pensés comme des petites collectivités plutôt homogènes (la famille, le groupe de rmistes, les jeunes délinquants, les jeunes sans emploi et peu qualifiés etc.). La solidarité n'est alors envisagée que dans sa dimension sociale et, à l'exception de quelques allusions au rôle du voisinage, rien n'est dit du territoire, ni dans l'interprétation des processus qui conduisent à l'exclusion sociale, ni a fortiori quand il s'agit d'avancer des préconisations.

L'intérêt de l'approche de l'exclusion par le territoire

Il y a donc bien un paradoxe dans l'opposition symétrique de ces deux positions: l'entrée par l'exclusion sociale ignore l'espace de vie et les territoires des individus et des groupes exclus, elle ne fait jamais référence à la territorialisation des politiques de lutte contre l'exclusion; la mise en évidence des ségrégations sociales dans l'espace est réductrice dans l'appréhension des processus individuels et sociologiques de l'exclusion, elle est au cœur du débat sur «les territoires contre l'exclusion ». Il nous paraît essentiel de dépasser cette contradiction en repensant la juste place du territoire dans les mécanismes de l'exclusion.

La condition préalable est de se démarquer des discours médiatiques et politiques qui associent, sans argumentation, fracture sociale et quartiers défavorisés ou ethniques. Les concepts dont la géographie dispose pour traiter de la relation espace/pauvreté, et en particulier celui de ségrégation spatiale, méritent d'être revisités face aux nouvelles formes d'exclusion ubiquistes que la société post-fordiste secrète. Le concept de territoire ne doit pas être posé en a priori, à l'amont de l'identification du mécanisme de l'exclusion. Il nous paraît important de faire l'hypothèse qu'il s'agit bien d'un facteur actif, sous-estimé dans l'approche sociologique, mais dont il convient de découvrir l'importance et la spécificité par le travail de recherche lui-même.

17 Cette hypothèse qu'il existe, pour la connaissance comme pour l'action, un enjeu dans la réintroduction heuristique du territoire dans les recherches sur l'exclusion est une hypothèse forte pour les chercheurs de STRATES et de la SEGESA engagés dans cette recherche L'exclusion en milieu rural. On peut avancer plusieurs arguments pour étayer cette position.

La mise à jour de l'effet spatial dans les processus de l'exclusion

18 À partir de la connaissance des types d'espaces ruraux, qu'ils soient définis comme des types structurels (espaces ruraux périurbains, espaces ruraux à fonction agricole, industrielle ou touristique dominante, espaces ruraux de faible densité...) ou par rapport à des risques de rupture (espaces ruraux fragiles ou très fragiles, espaces 
stables, espaces émergents...), on peut faire l'hypothèse que les transformations du territoire entraînent l'exclusion d'individus et de groupes.

Comme l'a montré Jean-Claude Bontron, la restructuration des activités productives, en particulier en agriculture, la rupture de la reproduction du travail familial (succession père/fils) et de la transmission ont entraîné une précarisation de l'emploi agricole et une paupérisation qui concerne aussi bien les chefs d'exploitation que les salariés agricoles. L'internationalisation des économies a accentué les risques de "faillite » (réforme de la PAC et politique des quotas, répercussions sur les prix et sur la consommation de la maladie de la vache folle, etc.) et donc d'exclusion par l'emploi.

Ce constat peut être transposé aux artisans et petits commerçants dont les revenus sont en baisse dans les espaces où se développent la concurrence des grandes surfaces. De même, lorsque l'industrie est présente dans les zones rurales, elle n'échappe plus au mouvement de restructuration et de régression de l'emploi industriel et à la crise, ce qui conduit à des processus d'exclusion assez spécifiques, compte tenu de la faible mobilité géographique et professionnelle et de l'étroitesse du marché.

Il peut donc être pertinent de tester l'hypothèse que le territoire dans sa dimension économique peut avoir un rôle actif dans le processus d'exclusion d'individus et de groupes sociaux. Ce type de relation société/territoire, la relation entre le système productif et le type d'espace, est généralement sous-estimé. La territorialisation de l'emploi et de son évolution (bilan création/disparition d'entreprises, offre d'emploi/ demande d'emploi/chômage, suivi des licenciements, etc.), peut apporter des pistes d'analyse des catégories sociales touchées par l'exclusion, à condition qu'elle se fasse à des niveaux locaux fins (qu'il s'agisse de quartiers urbains ou ruraux), sans recourir aux catégories spatiales définies a priori (catégories statistiques) ou sans réifier les types d'espaces qui ont été élaborés pour d'autres objectifs (types d'espaces fragiles, espaces dépendants de pôles urbains, etc.) ${ }^{6}$. Les faits d'excès peuvent aussi être des faits de territoire. On peut en tous cas au moins poser la question de la pertinence de l'approche territoriale pour analyser et repérer les phénomènes d'exclusion.

La mise en évidence par le territoire des effets croisés ou cumulés des processus

On peut aussi faire l'hypothèse que l'entrée par le territoire peut permettre de repérer d'éventuelles interactions entre des processus d'exclusion connus et analysés séparément et dont on ignore les relations de causalité. Ainsi, quelles sont les conséquences de l'aggravation du chômage et de la réduction de l'offre de travail non qualifiée sur toutes les catégories «traditionnelles » d'exclus (les handicapés physiques et mentaux, les délinquants mineurs, les prisonniers, les « gens du voyage »...) dont on préconisait souvent la réinsertion par le travail manuel (foyers de rééducation, CAT, activité de fabrication dans les prisons...) ? L'analyse dans un territoire précis des répercussions en dominos de la dégradation de l'offre d'emploi qualifié a également de l'intérêt : la progression du RMI dans des catégories sociales dont on ne soupçonnait pas le risque d'exclusion du travail ou de paupérisation (agriculteurs modernisés, jeunes diplômés, cadres moyens...) conduit-elle à une aggravation des possibilités d'insertion pour les catégories de travailleurs à plus faible qualification? quelles sont les conséquences de l'aggravation de la crise de l'emploi sur les migrations des individus et des familles en grande difficulté ? l'arrivée de citadins "exclus » à la campagne est-elle une réalité ? aggrave-t-elle la situation des exclus autochtones ? C'est au niveau local (départemental au maximum) qu'il serait possible de comprendre ces téléscopages de processus, de vérifier si la tendance est à l'aggravation ou au recul 
de l'exclusion dans un contexte précis, d'évaluer les possibilités de mener de front des politiques de lutte contre l'exclusion qui visent des publics d'exclus différents et qui peuvent donc être contradictoires, de mesurer l'effet global de ces politiques.

L'approfondissement du vécu individuel de l'exclusion en fonction des lieux de vie et des modes d'habiter

Il nous semble qu'il y a sous-estimation du rôle des espaces et des lieux de vie dans le vécu et dans le processus individuel de l'exclusion.

Notre hypothèse est que la qualité du milieu de vie peut agir (en accentuant ou en atténuant) le sentiment d'exclusion des individus et des groupes. Ainsi, en milieu rural, la désorganisation des services, l'éloignement progressif des équipements et des services, l'augmentation des prix qui résultent de certaines privatisations, risquent de poser problèmes à certaines catégories sociales, surtout les moins mobiles comme les personnes âgées ou les jeunes dont les parents sont eux-mêmes en difficulté.

De même, la plus ou moins grande désintégration de la cellule familiale, qui jouait un rôle important en milieu rural mais aussi dans certains milieux ouvriers, pourrait accentuer les difficultés des plus jeunes en particulier, mais aussi des femmes célibataires ou des divorcés. La famille rurale est le plus souvent géographiquement éclatée, avec un accroissement des ménages de une personne, en majorité des personnes âgées isolées. Cet éclatement de la famille a des conséquences sur la régulation des formes de déviance et de délinquance qui accompagne souvent l'« excès » de l'exclusion.

Les rapports de voisinage, souvent associés à des rapports solidaires ou communautaires, sont un facteur territorial et peuvent également jouer un rôle. Dans le quartier ou dans le village, ces relations, quand elles existent encore ou se reconstruisent, permettent la prise en charge des personnes en difficulté ou peuvent favoriser la lutte personnelle des individus contre l'incertitude de l'avenir. Mais ce rôle peut être surestimé, et les sociétés dite d'interconnaissance sont parfois plus excluantes que les sociétés de l'anonymat.

D'une façon générale, la crise de l'emploi, la peur de la régression sociale et de l'exclusion, poussent les individus à privilégier le mode d'habiter rural qui représente dans l'imaginaire ou réellement un territoire où l'on peut vivre modestement avec les avantages de la nature et de l'interconnaissance. La mobilité géographique est de plus en plus un moyen réel ou rêvé d'échapper à l'exclusion, les communes rurales devenant terres d'accueil pour exclus citadins.

28 L'hypothèse est donc que le territoire concret ou rêvé a une place dans la trajectoire individuelle de l'exclusion.

L'approfondissement des réactions individuelles en relation avec les types de milieux

On sait que les événements et les situations à l'origine de l'exclusion engendrent des réactions diverses des individus: détresse et dépression, auto-exclusion, résistance, recours à l'aide, initiative... Mais on sous-estime le fait que la spécificité de ces réactions individuelles va de pair avec les spécificités des milieux et espaces géographiques dans lesquels les individus vivent.

30 Cette hypothèse s'appuie sur l'observation des différences dans le mode de résistance des « exclus » selon les espaces. Dans les métropoles, avec l'aggravation de la pauvreté et de la misère s'est développée la presse de rue vendue pour permettre de " payer la chambre d'hôtel » et « de se laver » " pour trouver du travail ». Le phénomène des SDF 
est d'autant plus présent que la ville est de grande taille. Aux parvis d'autrefois se sont substitués les métros et RER, les entrées de grands magasins et hypermarchés pour ceux qui mendient, font de la musique ou des marionnettes, vendent des produits peu coûteux (objets fabriqués avec des tickets de métro par exemple). En milieu rural au contraire, la résistance s'effectue le plus souvent sur la base de la petite exploitation de subsistance à partir de laquelle on pourra retrouver une couverture sociale (Fabre, 1996), sur le jardin potager et l'autoconsommation, sur le travail précaire et saisonnier (vendanges, bûcheronnage, travail dans le bâtiment) et l'économie informelle.

31 Là encore, à condition de ne pas poser le territoire comme un facteur a priori de l'exclusion, il peut être intéressant de tester la valeur de cette hypothèse.

L'approfondissement des ressorts de l'action collective

32 La place du territoire dans la lutte contre l'exclusion est objet de controverse. D'un côté comme nous l'avons vu, le territoire est pensé comme le moyen pertinent et efficace pour combattre le chômage et la fracture sociale. Qu'il s'agisse de l'espace urbain (Développement Social de Quartier) ou de l'espace rural (La lutte contre l'exclusion dans les territoires ruraux, Brunet et al., 1996), l'action territoriale et le développement local sont considérés comme des démarches favorisant l'« insertion durable ». Cette position est dans le droit fil de la décentralisation et de la création de toutes les institutions dont la transversalité est appuyée sur la notion de territoire et de local (missions locales, associations de pays et intercommunalité, comités de bassins d'emploi, etc.).

De l'autre côté, se manifeste une certaine perplexité à l'égard de ces outils territoriaux qui ont plutôt débouché sur la stigmatisation de territoires comme celui des banlieues (ce qui est une forme d'exclusion) tandis qu'est privilégiée l'action sociale sur les individus et les catégories.

Les approches territoriales de la pauvreté et de l'insertion (en particulier de la politique du RMI et des comportement des rmistes (Balley et al., 1992, Géoforum 93, 1994) ont montré l'inégalité selon les territoires de la politique d'insertion. Il faut interroger ces disparités qui peuvent tenir à des différences de représentations et de pratiques de la lutte contre l'exclusion entre les différents partenaires. En ville comme à la campagne la volonté des associations, des collectivités locales et de certains organismes publics de lutter contre le chômage et l'exclusion par le territoire ("Territoires contre l'exclusion») va s'affirmant. L'hypothèse de l'efficacité des collectifs territoriaux mérite d'être évaluée.

En définitive, les enjeux de l'approche territoriale de l'exclusion sont à la fois des enjeux de connaissance $-\mathrm{y}$ a-t-il des spécificités d'occurrence de l'exclusion, des profils d'exclus, selon les types d'espaces? y a-t-il aggravation des processus individuels selon les territoires?-, mais aussi des enjeux d'action: quelle est réellement la place du territoire et de l'action locale dans la lutte contre l'exclusion et le processus inverse de l'insertion? 


\section{BIBLIOGRAPHIE}

ASSOCIATION DES RURALISTES FRANCAIS (1986), La pauvreté dans le monde rural, (Pierre MACLOUF, dir.), Paris, ARF/L'Harmattan, 329 p.

BALLEY Chantal, LENORMAND Pierre, MATHIEU Nicole (1991), Le RMI en milieu rural. Étude réalisée dans six départements, Paris, STRATES, $154 \mathrm{p}$.

BALLEY Chantal, LENORMAND Pierre, MATHIEU Nicole (1992), « Territoire rural, RMI, pauvreté », Sociétés contemporaines, 9, pp. 53-75.

BOURDIEU Pierre, dir. (1993), La Misère du monde, Paris, Seuil, 948 p.

BRUN Jacques, RHEIN Catherine, dir. (1994), La Ségrégation dans la ville, Paris, L'Harmattan, 262 p. (en particulier les articles de : BRUN Jacques, « Essai critique sur la notion de ségrégation et son usage en géographie urbaine », pp. 21-58 ; GRAFMEYER Yves, « Regards sociologiques sur la ségrégation », pp. 85-118; RHEIN Catherine, «La ségrégation et ses mesures », pp. 121-162 et « La division sociale de l'espace parisien et son évolution 1954-1975 », pp. 229-260.)

CASTEL Robert, LAÉ Jean-François (1992), Le Revenu Minimum d'Insertion. Une dette sociale, Paris, L'Harmattan.

CASTEL Robert (1995), Métamorphose de la question sociale. Une chronique du salariat, Paris, Fayard.

ENGELS Fiedrich (1845), La Misère des classes laborieuses en Angleterre, Trad. fr. 1933.

FABRE Camille (1996), « Ménages en situation de précarité et activité agricole. Analyse à partir d'études de cas réalisées en Haute Loire ", Agricultures, à paraître.

GÉOFORUM 93, «A la découverte des pauvretés et exclusions, Regards croisés », 1994, Géographes associés, 14-15, 182 p. (en particulier les articles de : BALLEY Chantal, « Éditorial », p. 7 ; BEHAR Daniel, « Poches d'exclusion ou fragmentation urbaine. Quels enjeux pour la politique de la ville?», pp. 67-72 ; GUILLOU Jacques, « L'errance des jeunes sans domicile fixe au sein des espaces publics », pp. 127-136 ; JAILLET Marie Christine, « La territorialisation des politiques de lutte contre l'exclusion ", pp. 63-66 ; MESINI Béatrice, Le paradigme de l'exclusion : dynamique et rétroaction du territoire local, pp. 113-118; et une importante bibliographie pp. 170-174.)

JOINT-LAMBERT Marie-Thérèse (1995), « Exclusion : pour une plus grande rigueur d'analyse », Droit social, 3, mars, pp. 215-221.

MATHIEU Nicole (1991), « La pauvreté en milieu rural », Alternatives économiques, 12, pp. 12-14 (supplément « Pauvreté, pauvretés »).

MATHIEU Nicole (1992), « Ruralité et pauvretés », Sève, 542, nov., pp. 557-565 (Dossier « État de la France rurale $»)$.

MOREAU de BELLAING Louis (1996), «L'exclusion : des mots pour le dire », Communication au Colloque « Exclusion, Psychiatrie, Travail social », 24 sept. 1996, 16 p.

PAUGAM Serge (dir.) (1996), L'Exclusion - L'état des savoirs, Paris, La Découverte, 582 p.

SASSIER Philippe (1996), «Évolution des représentations de l'exclusion », Communication au Colloque « Exclusion, Psychiatrie, Travail social », 24 sept. 1996, 8 p.

TOUTAIN Xavier, KENNY-CAZIN Anne, MATHIEU Nicole (1996), L'exclusion en milieu rural, Rapport final, Paris, SEGESA/STRATES, 84 p. + annexes et bibliographie (Contrat Ministère du Développement Économique et du Plan). 


\section{NOTES}

1. Cf. l'article «Exclusion » dans Campagnes solidaires, mars 97, 106, pp. 8-9 : « Face à la montée de la précarité, trente-trois associations engagées contre les exclusions, dont Solidarité paysans, ont élaboré un texte "Propositions contre la précarisation et les exclusions" (contact : Muriel Rozenfeld, tél : 01449214 32) visant à rétablir les droits sociaux fondamentaux affirmés par le préambule de la Constitution de 1946. Ceci, afin que la loi-cadre actuellement préparée par le gouvernement, qui doit faire l'objet d'un prochain débat à l'Assemblée nationale, traduise réellement la volonté solidaire de la collectivité nationale. "

2. Cf. le FIG de Saint-Dié 1996.

3. Le modèle de l'Amérique du Sud et des PVD est plus complexe car on observe une coexistence de ces deux modèles.

4. Dérive qui frappe même les acteurs spécialisés du développement rural qui vont directement au " rôle de l'intercommunalité » pour lutter « contre l'exclusion dans les territoires ruraux ». Cf. Caisse des Dépôts et Consignations, Mairie-Conseils, Programme de Développement Rural, Programme Développement Solidarité, Fédération des Parcs Naturels Régionaux de France, 1996, La lutte contre l'exclusion dans les territoires ruraux. Le rôle de l'intercommunalité, Paris, La Documentation française, $123 \mathrm{p}$.

5. «L'exclusion, la désinsertion, le chômage, l'abandon, la désaffiliation, la vulnérabilité, la désocialisation, la sous-prolétarisation, la désidentification la déculturation, (sont des) processus qui peuvent être analysés comme des faits sociaux marqués par l'excès. " (Moreau de Bellaing, 1996).

6. Ainsi la catégorie de « rural » qui figure au point de départ de cette recherche, de même que la question de la dichotomie rural/urbain qui lui est sous-jacente (y a-t-il des formes d'exclusion en milieu rural qui seraient foncièrement différentes de l'exclusion urbaine ?) ne sont que des catégories provisoires pour poser la question d'éventuelles spécificités ou intensité des processus d'exclusion selon les spécificités de formes spatiales identifiées et singulières. Autrement dit, les types d'espaces ruraux dans lesquels la recherche est menée sont des catégories temporaires pour interroger les processus et les profils de l'exclusion, mais ils n'ont pas de valeur a priori pour définir des spécificités en relation avec des faits de territoire, ces derniers ne devant être déterminés qu'à l'aval de la recherche. L'énoncé de la diversité des relations exclusion/ territoire est donc un résultat escompté à son terme.

\section{RÉSUMÉS}

Les rapports entre pauvreté, culture et territoire ont donné lieu à des travaux théoriques importants en géographie comme en sociologie. Mais ces théories sont-elles pertinentes pour traiter des phénomènes d'exclusion d'aujourd'hui, de plus en plus associés à la crise de l'emploi et aux changements des représentations du travail salarié ? Cet article invite les chercheurs à se poser radicalement la question de cette pertinence en proposant des pistes pour entrer dans la complexité des relations exclusion/territoire. 


\section{Social exclusion : the spatial approach revisited}

The relations between poverty, ethnicity and territory have been analysed in famous theoretical publications coming from sociologist as well as geographers. But are these theories able to help us to understanding and resolving what is call "exclusion» today? This paper focuses on the answer, investigating the various ways to identify and schematize the links between exclusion and spatial analysis.

\section{INDEX}

Mots-clés : exclusion, crise de l'emploi, territoire, théories sociales, théories spatiales Keywords : territory, social theories, spatial theories

\section{AUTEUR}

\section{NICOLE MATHIEU}

Nicole MATHIEU est directeur de recherches au CNRS (LADYSS). Avec une spécialisation de géographie rurale et sur les problèmes dits d'environnement, elle s'attache à promouvoir une conception de la recherche donnant une valeur à l'expression de la subjectivité, aux pratiques collectives, à l'interdisciplinarité. Sa démarche privilégie un va et vient entre différents niveaux (analyses locales, analyses générales en France et en Europe). 